Aus der Universitätsklinik für psychische und nervöse Krankheiten in Gießen.

\title{
Die scheinbaren elektrischen Ladungen des menschlichen Körpers.
}

Von Prof. Dr. Sommer in Gießen und Robert Fürstenau in Berlin.

Die Entstehung der Elektrizitätsmengen, welche die bei dem Reiben eines Kompasses oder eines evakuierten Glasgefäßes auftretenden Phänomene hervorrufen, läßt sich theoretisch auf zwei Ursachen zurückführen: entweder entsteht die wirksame Ladung durch die Reibung des Fingers am Glas, oder aber der Finger besitzt, wenn vielleicht auch nur unter gewissen Umständen, an seiner Spitze eine freie elektrische Ladung, welche auf das Glas bei der Berührung übertragen wird. Pfaff ${ }^{1}$ ) spricht in seinen Arbeiten stets von einer solchen freien Ladung; so sagt er z. B.: „Reizbare Menschen von sogenanntem sanguinischen Temperament haben mehr

1) Pfaff, Meckels deutsches Archiv für Physiologie Bd. 3, 1817. 
freie Elektrizität als träge von sogenanntem phlegmatischen Temperament. - Des Abends ist die Menge der Elektrizität größer als zu andern Tageszeiten. - Geistige Getränke vermehren die Menge der freien Elektrizität."

Harnackl) weist in seinen Kompaßversuchen von neuem auf diese vermeintlichen elektrischen Ladungen der Finger hin. Er ist der. Meinung, da $B$, um die elektrischen Phänomene hervorzurufen, die Reibung nebensächlich, ja unter Umständen entbehrlich sei; es werde nicht etwas erzeugt, sondern es sei bereits etwas vorhanden. Dieses bereits Vorhandene wird bei der Berührung nach seiner Meinung auf das Glas übertragen; auffällig ist es nun aber doch, $\mathrm{da} B$ irgend welche Effekte nie ohne mehrmaliges Streichen hervorgebracht werden können. Als beweiskräftige Tatsache für seine Ansicht führt Harnack an: „Das jeweilig zu leistende Quantum wurde schon nach wenigen Streichungen erreicht, das Elektrometer blieb dann auf dieser Grenze stehen, und man mochte fortreiben, so viel man wollte - es gelang nicht, die Ladung weiter zu erhöhen. Die Fingerspitze gab also ihr Quantum ab (!), mochte es viel oder wenig sein, und alles weitere Reiben fruchtete dann nichts mehr."

Die Fingerspitze hat also hiernach von vornherein eine freie elektrische Ladung und gibt diese an die geriebene Glasfläche ab, wird das Streichen jedoch weiter fortgesetzt, so sinkt der Ausschlag des Elektrometers, der Finger leitet jetzt also die Ladung, die er eben abgegeben hatte, wieder ab. Das ist doch ein recht unwahrscheinlicher Vorgang; denn warum leitete der menschliche Körper nicht schon vorher dieses an der Fingerspitze vorhandene Quantum ab? Oder tat er es, und wurde stets eine neue Elektrizitätsmenge an der Fingerspitze erzeugt? Warum sollte dann aber diese Erzeugung plötzlich nach kürzerem oder längerem Reiben des Glases aufhören? Harnack widerspricht sich auch selbst und seiner Auffassung, wenn er als Stütze derselben anführt: „Hatte ich die Glasplatte gerieben, so wirkte die (geriebene) Glasstange bei der Annäherung an das Elektroskop (ohne Berührung) steigernd, die Lackstange schwächend; war die Hartgummiplatte gerieben worden, so verhielt sich die Sache umgekehrt." Harnack zieht die richtige Folgerung daraus: Durch die Streichungen mit der Fingerspitze war demnach die Glasplatte positiv, die Hartgummiplatte negativ geladen worden."

Es ist also das eingetreten, was stets erfolgt, wenn man eine Glas- oder Hartgummiplatte etwa mit einem Stück Seide reibt. In welchem Widerspruch steht aber dieser Versuch zu der Auffassung der freien Ladungen an den Fingerspitzen! Wenn die letzteren wirklich eine Ladung besäßen, so könnte diese doch nur ein ganz bestimmtes, nicht wechselndes Vorzeichen besitzen, und nicht einmal positiv, einmal negativ sein, je nachdem man die Glasplatte streicht oder die Hartgummiplatte. Hätte aber die Fingerspitze eine nach ihrem Vorzeichen eindeutig bestimmte Ladung, etwa eine positive, so müßte bei Streichen der Glasplatte ein bei weitem größerer Ausschlag am Elektroskop zu beobahten sein, als beim Streichen der Hartgummiplạtte, da letztere durch das Reiben selbst negativ elektrisch wird, und diese negative Elektrizität die positive des Fingers binden würde.

Die am Elektroskop bei Annäherung der Finger beobachtete Anziehung und Abstoßung der Blättchen ist, wie an anderer Stelle ${ }^{2}$ ) schon nachgewiesen, nicht eine Folge von frei existierenden Ladungen an den Fingern, sondern nichts anderes als eine auf den Finger vom geladenen Elektroskop ausgeübte Influenzwirkung, die im allgemeinen eine Anziehung zwischen Finger und Elektroskopblättchen zur Folge hat; eine AbstoBung tritt nachgewiesenermaßen ein, wenn a u B rdem noch auf der Glaswand des Elektroskopes Ladungen vorhanden sind.

Ein Experimentum crucis für das Vorhandensein von statischen Ladungen an den Fingerspitzen wäre nur das eine: wenn nämlich ein vollständig entladenes Elektroskop bei Berührung des Metallknopfes mit dem menschlichen Finger einen Ausschlag geben würde. Sommer, der entgegengesetzt der Pfaff schen A uffassung die scheinbaren Ladungen des Elektroskops durch die Finger rein physikalisch aus einer gleichzeitigen Ladung der Glashülle erklärt hat, machte nachträglich einige Beobachtungen, die dieser eigenen Erklärung $\mathrm{zu}$ widersprechen und die $\mathrm{P}$ faff-Harnacksche Ansicht zu bestätigen schienen. Weun eine Versuchsperson mit der Influenzmaschine gearbeitet hatte und nach längerer Zeit den Metallknopf des Elektroskopes berührte, so zeigte sich fast stets ein merkbarer Ausschlag der Aluminiumblättchen. Ja, selbst wenn das Elektroskop tagelang unberührt, fern von jeder Quelle statischer Elektrizität, gestanden hatte, und von Personen, die mit elektrischen Apparaten gearbeitet hatten, berührt wurde, so erhielt man öfter einen durchaus merkbaren Ausschlag. Diese Phänomene

1) Harnack, Studien über Hautelektrizităt und Hautmagnetismus des Menschen. Jena 1905. - 2) So m mer, Elektromotorische Wirkungen der Finger. Neurologische Zentralblatt 1905 , No. 7 . deuteten zunächst auf eine tatsächlich vorhandene freie elektrische Ladung des menschlichen Körpers. Bei genauerer Nachprüfung dieser Versuche haben wir jedoch gefunden, da $\beta$ diese Ladungen der Finger nur scheinbare sind, genau wie bei den erwähnten Experimenten Sommers.

Wir stellten zunächst fest, dafß es für die Person desjenigen, der das Elektroskop berührt, durchaus gleichgültig ist, ob er mit der Influenzmaschine gearbeitet hat oder nicht; der Erfolg tritt sogar ebensogut ein, wenn man den Elektroskopknopf nicht mit dem Finger, sondern mit einem zur Erde abgeleiteten Metallgegenstand, etwa einer Messingkette, berührt. Es ist sogar zu bemerken, daß, wenn man das Elektroskop einen Augenblick mit dem Finger und kurz darauf mit der Kette in Verbindung bringt, der durch die Berührung des Fingers erhaltene Ausschlag sich bei der zweiten Berührung noch vergrößert. Hätte der Finger ein Elektrizitätsquantum auf das Elektroskop gebracht, so hätte die Kette es wieder notwendigerweise entfernen müssen und nicht noch etwas hinzufügen konnen.

Es ist vielmehr unumgänglich anzunehmen, da $\beta$ es sich lediglich um Influenzerscheinungen handelt. Das Elektroskop hatte während der eben beschriebenen Versuche in nicht allzu großer lintfernung von der Influenzmaschine gestanden, und diese war stets vor der Berührung des Elektroskopes etwa eine halbe Minute lang in Tätigkeit gesetzt worden. Dadurch hatte die Glaswandung des Elektroskopes eine Influenzladung angenommen, welche eine Verteilung der Elektrizität im Innern des Elektroskopes hervorrief. Wurde nun durch die Berührung des Elektroskopknopfes die gleichnamige Elektrizität zur Erde abgeleitet, so mußte notgedrungen eine elektrostatische Anziehung zwischen Glaswand und Aluminiumblättchen resultieren, soda $B$ es den Anschein erwecken konnte, als habe die Hand dem Elektroskop eine Ladung verliehen. Eine einfache Probe für die Richtigkeit dieser Erklärung ist die, daß man, während die Influenzmaschine erregt wird, die Glaswandung zur Erde ableitet, und so das Ansammeln einer Ladung auf ihr verhindert. Man wird dann höchstens bei ganz trockener Luft einen Ausschlag erhalten, dagegen nie bei feuchtem Wetter, wobei die Luftfeuchtigkeit sich an der Entladung der Glaswand beteiligt.

Die Vermutung liegt nahe, daß auch dann eine, wenn auch nicht bemerkbare, Ladung auf der Glaswand vorhanden gewesen war, wenn man das scheinbar ganz entladene Elektroskop, das nicht irgend welchen elektrischen Wirkungen ausgesetzt war, berührt und einen Ausschlag erhalten hatte. Entweder konnte der Glaswandung des Elektroskopes vorher durch irgend einen Zufall eine Ladung influenziert worden sein, oder aber, sie hatte sich durch atmosphärische Einflüsse von selbst aufgeladen, jedoch stets in so schwachem Maße, daß an den Blättchen keine Spur von dem Vorhandensein einer solchen Ladung zu bemerken war.

Um die erste Möglichkeit zu prüfen, ließen wir das Elektroskop eine halbe Minute in etwa $2 \mathrm{~m}$ Entfernung von der während derselben Zeit in Betrieb gesetzten Influenzmaschine stehen. Die Blättchen zeigten nach Aufhören des Arbeitens mit der Maschine keine Spur eines Ausschlags. Dann wurde das Elektroskop, welches auf einen Paraffinklotz gestellt war, damit während des Transportes die vermutliche Ladung nicht verloren ging, weit von der Maschine entfernt und geprüft, wie lange die Ladung auf der Glaswand verblieb. Wenn man nach 10 Minuten den Knopf des Elektroskopes berührte, war zuweilen ein Ausschlag der Blättchen bis zur Glaswand zu beobachten, oft auch nur ein solcher von 5 bis 10 Bogengraden. Wenn noch länger gewartet wurde, war ein Ausschlag nur selten noch festzustellen.

Wir stellten das Elektroskop, welches sorgfältig entladen war, in der Nähe der Dampfheizung auf; als wir es nach etwa zehn Minuten berührten, zeigten die Blättchen einen deutlichen Ausschlag; die Glaswand hatte also offenbar eine Ladung angenommen, vielleicht infolge der Luftströmungen an der Heizung. Das Elektroskop wurde in anderen Räumen aufgestellt; stets war, wenn man den Knopf nach etwa einer Viertelstunde berührte, ein deutlicher Ausschlag zu bemerken.

Um endgültig zu prüfen, ob die Erscheinung ihre Ursache in einer Aufladung der Glaswand hatte, wurde letztere durch eine Kette zur Erde abgeleitet, während das Elektroskop sich in denselben Räumen befand wie vorher. Wenn entsprechend der $\mathrm{Har}$ nackschen Annahme der Finger bei Berührung sein während der Viertelstunde etwa wieder angesammeltes Elektrizitätsquantum an das Elektroskop abgäbe, so hätte man bei dieser Anordnung einen großen Ausschlag zu erwarten, da dann die von den Blättchen her bewirkte Verteilung und die Ableiting der gleichnamigen Elektrizität zur Erde eine beträchtliche Anziehung zwischen Blättchen und Glaswand zur Folge hätte haben müssen. In Wirklichkeit zeigte sich nicht der geringste Ausschlag, weil eben jedes Entstehen einer Ladung auf der Glaswand durch die Ableitung zur Erde verhindert war. Aus 
eben demselben Grunde mißlingen die Versuche stets bei sehr feuchter Wittering. Auch eine große Anzahl von Versuchspersonen vermochten, gleichgültig, ob sie mit der Influenzmaschine gearbeitet hatten oder nicht, keinen Ausschlag am Elektroskop zu erzielen, wenn die Glaswandung zur Erde abgeleitet war. Dieselben Versuchspersonen brachten die Blättchen jedoch zum Ausschlagen, wenn die Glaswand nicht abgeleitet war, und das Elektroskop einige Zeit ruhig in einem trockenen Raum gestanden hatte.

Es geht aus diesen Versuchen mit völliger Deutlichkeit hervor, daß die menschliche Fingerspitze an sich keine freie elektrische Ladung besitzt, wie sie sie nach physikalischen Voraussetzungen auch nicht besitzen kann.

So sind auch die Versuche über Leuchterscheinungen beim Reiben evakuierter Glasbirnen zu verstehen: es wird vom Finger keine Ladung auf das Glas übertragen, sondern lediglich statische Elektrizität durch Reibung erzeugt. Nur sind die Vorgänge in den Lampen wesentlich komplizierterer Natur als bei den Elektroskopversuchen. Es wurde schon in einer früheren Arbeit ${ }^{1}$ ) darauf hingewiesen, daß die Leuchterscheinungen, vor allem das Nachleuchten, sehr verschieden ansfallen, je nachdem man Lampen mit einem Kohlefaden benutzt oder einfache evakuierte Röhren ohne Elektroden. Es kommt dies daher, daß Ladungen an der inneren Glaswand der Glühlampen eine äußerst wichtige Rolle spielen.

Der Vorgang beim Reiben einer Lampe mit Kohlefaden ist folgender: Durch die Reibung mit der Hand wird die äuBere Glaswand der Lampe positiv elektrisch, was man am Elektroskop feststellen kann. Diese Ladung bewirkt auf der inneren Glaswand eine elektrische Verteilung. Nun wird durch den beim Reiben fortwährend gegen die Lampenwandungen schlagenden Kohlefaden die gleichnamige Elektrizität zur Erde abgeleitet, und es bleibt auf der Innenseite der Lampe eine negative Ladung. Daß diese wirklich vorhanden ist, haben wir folgendermaßen nachgewiesen: Nachdem wir die Lampe einige Zeit gerieben hatten, entfernten wir die auf der äußeren Glaswandung haftende positive Elektrizität und verbanden die Fassung der Lampe mit dem Knopf des Elektroskops. Wir faßten dann die Lampe am Glase an, nicht an der Fassung, und schüttelten sie, sodaß der Kohlefaden in Schwingungen geriet. Im Moment nun, wo er die innere Glaswand berührte, zeigte das Elektroskop einen sehr großen Ausschlag, der durch Prüfung mit einem geriebenen Glas- und Hartgummistab als negativ festgestellt wurde.

Daher erklären sich auch die im ersten Augenblick ein wenig rätselhaften Leuchterscheinungen, welche auftreten, wenn man die Lampe schüttelt oder schlägt, nachdem sie ganz entladen erscheint. Oft kann man auch in geriebenen Lampen beobachten, daß der Kohlefaden sich nicht nur an die Glaswand anlegt, sondern sprung- und ruckweise an ihr entlang wandert; auch dieses Phänomen ist eine natürliche Folgeerscheinung dieser, ,inneren Ladung“. Sobald nämlich der Kohlefaden an einer Stelle die Elektrizität abgeleitet hat, wird er durch seine Spannkraft in die normale Lage zurïckgezogen, im selben Augenblick jedoch schon von einer der ersten benachbarten Stelle, die noch Ladung besitzt, angezogen, und so immer wieder von anderen Stellen, sodaß er also gezwungen wird, in der Lampe umherzuwandern.

Das Vorhandensein dieser Ladungen auf der Innenseite der Glaswand bedingt nun auch den ganz charakteristischen Unterschied im Ausfall der Versuche, wenn man statt der Glühlampen elektrodenlose Röhren benutzt. Da in letzteren nur eine Verteilung der Elektrizität auf der inneren Glaswand infolge Influenz von der äußeren her stattfinden kann, die gleichnamige Elektrizität jedoch nicht zur Erde abgeleitet wird, so befindet sich in elektrodenlosen Glasgefäßen ausschließlich auf der Außenseite eine positive elektrische Ladung. Sobald diese Ladung nicht mehr vorhanden ist, ist es auch unmöglich, weitere Entladungen in der Röhre zu erhalten. Wenn das Glas sehr schlecht leitet, wird die Ladung nur allmählich verschwinden, und aus diesem Grunde wird man dann ein kurz andauerndes Nachleuchten beobachten können, das indessen gar keine Aehnlichkeit mit den prächtigen Nachentladungen in den Glühlampen besitzt. Leitet das Glas jedoch gut, so wird die Röhre mit Aufhören des Reibens auch kein Leuchten mehr zeigen; auch am Elektroskop ist dann nirgends mehr eine Ladung nachzuweisen. Ganz anders verhalten sich in diesem Falle die Glühlampen. Leitet das Glas gut, sodaß eine äußere Ladung einige Zeit nach dem Reiben aicht mehr vorhanden ist, und berührt man jetzt mit der Lampe das Elektroskop, so zeigt dieses einen kräftigen Ausschlag, der indessen wieder zurückgeht, wenn man die Lampe entfernt. Er ist also nur durch Influenz von der auf der Innenseite der Lampe befindlichen, und wie man wieder prüfen kann, negativen Ladung her, entstanden. Diese Erscheinung ist nie an elektrodenlosen Röhren zu beobachten.

1) Fürstenau, Ueber Leuchterscheinungen beim Reibert evakulerter Röhren. iü
Die Versuche bestätigen die früher ausgesprochene Ansicht, $\mathrm{da} B$ die Leuchterscheinungen durch nichts anderes hervorgerufen werden, als durch eine Aenderung des elektrischen Feldes.') Beim Reiben der Lampen haben wir ein Anwachsen des elektrischen Feldes, verstärkt durch Anschlagen des Kohlefadens gegen die Glaswand: daher stärkere Leuchterscheinungen in Glühlampen als in elektrodenlosen Röhren. Nach Aufhören des Reibens kann spontan ein Aufleuchten stattfinden, da die änßere Glaswand ihre Ladung allmählich verliert; dies Leuchten kann nur ein mattes Schimmern sein, da das elektrische Feld sich nur langsam ändert. Und so ist es ja in der Tat zu beobachten. Ein spontanes Leuchten kann ferner auftreten, wenn der Kohlefaden infolge der elektrostatischen Anziehung sich gegen die innere Glaswand legt und so durch Ableitung hier eine Aenderung des Feldes hervorruft. Um vieles stärker wird das Leuchten, wenn man die Glaswand der Lampe berührt oder gar anhaucht, da dann die Aenderung des Feldes bedeutend schneller vor sich geht. Endlich, wenn außen keine Ladungen vorhanden sind, haben wir noch im Innern der Lampe ein starkes elektrisches Feld, das sich ändert, wenn der Kohlefaden durch Schütteln oder Schlagen mit der Glaswand in Berührung gebracht wird: daher die Erscheinungen des Nachleuchtens.

Wir sehen also überall immer nur ein Entstehen und Verschwinden von Elektrizität, ein Aufladen und Entladen; von vornherein vorhanden ist nichts. Freie Ladungen an der Körperoberfläche existieren nicht. Daher ist der Grund für den verschiedenen Ausfall der Versuche mit verschiedenen Personen nicht in einer individuell wechselnden Quantität freier Elektrizită zu suchen, sondern in andern rein physikalischen Umständen, die freilich ihre physiologischen Ursachen haben können.

Der Streit über die physiologische oder physikalische Natur der Erscheinungen ist nichts als ein unnützer Wortstreit. Die physikalische Natur aller Erscheinungen steht doch außer Frage, und die physiologischen Momente, welche modifizierend auf die Vorgänge einwirken, sind ihrerseits doch wiederum in ihren Wirkungen rein physikalischer Natur. Oder ist die durch psychische und physiologische Einflüsse wechselnde Menge der Schweiß sekretion, die bei den Versuchen eine große Rolle spielt, nicht dadurch, daß sie die Leitfähigkeit der Haut ändert, ein rein phy sikalisch wirkendes Endprodukt von Ursachen, die ihrerseits freilich in physiologischen Vorgängen im Menschen liegen?

Es kommt als wesentlicher Faktor die Art des Reibens hinzu: wenn man ein intensives Leuchten erzielen will, so muß man nicht gleichmäßig reiben, sondern mehr schnellende Bewegungen ausführen, und die Bewegungen plötzlich unterbrechen, wie Sommer dies schon in seiner ersten Mitteilung hervorgehoben hat. $\left.{ }^{2}\right)$ Je schneller nämlich eine Aenderung im elektrischen Feld vor sich geht, desto kräftiger sind die erzielten Wirkungen. $\mathrm{Zu}$ einer solchen Bewegungsart ist nun bei weitem nicht jeder Mensch geeignet, wie man leicht beobachten kann; viele sind garnicht imstande, eine solche plötzliche Unterbrechung einer Bewegung mit Aenderung der Richtung fertig zu bringen.

Außer diesem Umstande spielt aber, wie schon bemerkt, die Schweißsekretion eine sehr große Rolle. Es ist von anderer Seite darauf hingewiesen worden, $\mathrm{da} B$ die Aenderungen derselben doch nur sehr geringe, kaum bemerkbare sind, und daß solche geringen Schwankungen doch unmöglich die großen Differenzen in dem Ausfall des Leuchtens erklären können. Wir treten entschieden dieser Ansicht entgegen. Man vergleiche die Haut mit dem ihr in bezug auf die elektrischen Phänomene in mancher Beziehung ähnlichen Glas. Wenn das Glas, im trockenen Zustande ein ausgezeichneter Isolator, nur von einer unmeßbar dünnen Flüssigkeitshant bedeckt ist, so stellt es einen guten Leiter dar, und die geringsten Aenderungen in der Menge der Feuchtigkeit rufen wesentliche Differenzen in der Leitfähigkeit hervor.

Wie gerade die Leitfähigkeit der Haut infolge der wechselnden Schweißsekretion von Stunde zu Stunde bei demselben Individuum in verblüffendem Maße wechselt, ist klar zu erkennen aus Versuchen über die bei Berührung von Metallelektroden mit der Haut auftretenden elektrischen Ströme, welche in Kürze an anderer Stelle veröffentlicht werden. ${ }^{3}$ ) Gerade die Leitfähigkeit der Haiut spielt auch bei den Reibungsversuchen eine große Rolle; es treten nämlich bei der Reibung noch kompliziertere Verhältnisse auf, wie sie oben nur schematisch entwickelt sind. Es sind wiederum Influenzwirkungen von der inneren Glaswand auf die äußere nachweisbar, und man kann sich nun leicht vorstellen, daß es darauf

1) Sommer, Die Natur der elektrischen Vorgănge an der Haut, besonders der Finger. Münchener medizinische Wochenschrift 1905 , No. 51 . - Fürstena u, Ueber einige Entladungserscheinungen in evakuierten Röhren. Annalen der Physik. Vlerte Folge 1905, Bd. 17. - 2) Vgl. Deutsche medizinische Wochenschrift 1905, No. 8: Lichterscheinungen nach Reibung der menschlichen Haut mit Glühlampen. - 3) Somme und Fürstenau, Die elektrischen Vorgänge an der menschlíchen Haut. Klinik für psychische und nervöse Krankheiten. Verlag von Marhold, Halle. 
ankommen muß, wie schnell auch von der äußeren Glaswand Ladungen, die von elektrischer Verteilung herrühren, abgeleitet werden, um wieder möglichst plötzliche Aenderungen im elektrischen Feld zu erhalten. Hierbei kommt die Haut mit ihrer doppelten Funktion als Leiter und Nichtleiter zur Wirkung. Deshalb erhält man auch durch Reiben der Lampen mit Pelzen oder Seide weitaus nicht die glänzenden Resultate wie bei dem Reiben mit der menschlichen Haut. Die Hautfeuchtigkeit hindert nicht das Zustandekommen der Leuchterscheinungen, sondern begünstigt sie. Hier vereinigen sich die Wirkungen der physiologisch bedingten Feuchtigkeit und der Bewegungsart. Wenn jemand, der einen bestimmten Grad von Hautfeuchtigkeit besitzt, nicht befähigt ist, während des Reibens die Bewegung kurz abzubrechen, so wird er, da die Elektrizität am Glas, wenn es etwas leitend ist, entlang wandert, die Ladung immer wieder entfernen, ohne daß er nennenswerte Leuchterscheinungen erhält. $\mathrm{Da}$ es sich ja im wesentlichen um Bewegung im elektrischen Felde handelt, ist es ganz zweifellos, daß unabhängig von der wechselnden Schweißsekretion einen großen Einfluß diese individuell verschiedene und wechselnde Bewegungsart besitzt. Hierin liegt entschieden ein physiologisches Moment, unabhängig von dem durchaus physikalischen Charakter der Erscheinungen. 